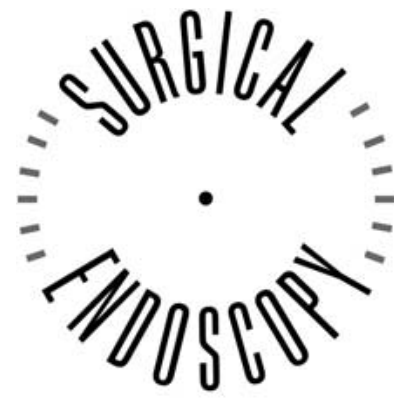

and Other Interventional Techniques

\title{
Quality-of-life outcomes with laparoscopic vs open cholecystectomy
}

\section{J. M. Quintana, ${ }^{1}$ J. Cabriada, ${ }^{2}$ I. Aróstegui, ${ }^{3}$ I. López de Tejada, ${ }^{4}$ A. Bilbao ${ }^{1}$}

${ }^{1}$ Unidad de Investigación, Hospital de Galdakao, Barrio Labeaga sn, 48960, Galdakao, Vizcaya, Spain

${ }^{2}$ Servicio de Digestivo, Hospital de Galdakao, Galdakao, Vizcaya, Spain

${ }^{3}$ Departamento de Matemática Aplicada, Estadistica e Investigación Operativa, Universidad del Pais Vasco, Lejona, Vizcaya, Spain

${ }^{4}$ Servicio de Cirugia, Hospital de Santiago, Vitoria, Alava, Spain

[Surg Endosc (2003) 17: 1129-1134, DOI: 10.1007/s00464-002-9202-2]

In the "Materials and methods" section, under the subheading "Statistical analysis," it was stated that just patients with ASA IV were in the high surgical risk group, whereas the high surgical risk group included patients with ASA III and ASA IV. 